\title{
Recurrent white thrombi formation in hemodialysis tubing: a case report
}

\author{
Kiran P Sathe', Wee-Song Yeo ${ }^{1}$, Isaac Desheng Liu', Sudha Ekambaram', Mohammed Azar', Hui-Kim Yap ${ }^{1,2 \dagger}$ \\ and Kar-Hui $\mathrm{Ng}^{1,2^{*}+}$
}

\begin{abstract}
Background: While the appearance of red clots in the dialyzer is a common phenomenon in every hemodialysis unit, the occurrence of white thrombi in the tubing is relatively rare.

Case presentation: We describe an adolescent male with recurrent white thrombi formation in the hemodialysis tubing. This patient had chronic renal failure from focal segmental glomerulosclerosis, but was no longer nephrotic at the time of the thrombi formation. He had a history of recurrent thrombosis of his vascular access. However, no pro-thrombotic risk factors could be identified. White particulate matter, measuring 1 to $3 \mathrm{~mm}$ in size, and adherent to the arterial and venous blood tubing lines was found during the rinse back of a hemodialysis session. This was associated with a $60 \%$ decrease in his platelet count. Light microscopic examination of the deposits revealed the presence of platelet aggregates. He subsequently developed thrombosis of his arteriovenous graft six hours later. The white thrombi recurred at the next dialysis session, as well as six months later. These episodes occurred regardless of the type of dialysis machine or tubing, and appeared to resolve with an increase in heparin dose.

Conclusion: Recurrent white thrombi formation can occur in the hemodialysis tubing of a patient with no identifiable pro-thrombotic factors. The white thrombi may be a harbinger of arteriovenous graft thrombosis and may be prevented by an increase in heparin dose.
\end{abstract}

Keywords: Platelet activation, Renal dialysis, Thrombosis, Blood platelets, Hemodialysis, Home

\section{Background}

Surface interaction between artificial material and blood during hemodialysis can lead to activation of complement, platelets as well as the coagulation cascade. The dialyzer membrane is often thought to be the main culprit in initiating these reactions, while the blood tubing in the circuit is generally believed to be inert. Biocompatibility of artificial materials used in hemodialysis is determined by the type of material, sterilisation mode and geometry [1,2]. Poor biocompatibility can lead to decreased platelet counts and abnormal platelet function during dialysis [3], but acute thrombotic events are rare.

\footnotetext{
* Correspondence: kar_hui_ng@nuhs.edu.sg

${ }^{\dagger}$ Equal contributors

'Shaw-NKF-NUH Children's Kidney Centre, Khoo Teck Puat-National University Children's Medical Institute, National University Health System, Level 12 NUHS Tower Block, 1E Kent Ridge Road, Singapore 119228, Singapore ${ }^{2}$ Department of Paediatrics, Yong Loo Lin School of Medicine, National University of Singapore, Singapore, Singapore
}

Here, we describe a case of recurrent white (platelet) thrombi formation in the hemodialysis tubing of a patient with no identifiable pro-thrombotic risk factors.

\section{Case presentation}

The patient is a 16-year old Indian male who presented at the age of two years with nephrotic syndrome. This did not respond to initial steroid treatment and renal biopsy eventually revealed collapsing focal segmental glomerulosclerosis. No mutations in podocin and nephrin genes were found. He responded to a combination treatment regime comprising of prednisolone, cyclosporine $\mathrm{A}$ and mycophenolate mofetil. After a course of rituximab at the age of 14 years, he achieved remission and was maintained without steroids or other immunosuppressive therapy. Unfortunately, he relapsed one year later and progressed quickly to end-stage renal failure when he was 16 years old.

He was commenced on thrice-weekly conventional hemodialysis, using Gambro ${ }^{\circ} \mathrm{AK} 95^{\mathrm{ma}}$, via a permanent

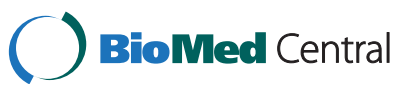

(c) 2015 Sathe et al.; licensee BioMed Central. This is an Open Access article distributed under the terms of the Creative Commons Attribution License (http://creativecommons.org/licenses/by/4.0), which permits unrestricted use, distribution, and reproduction in any medium, provided the original work is properly credited. The Creative Commons Public Domain Dedication waiver (http://creativecommons.org/publicdomain/zero/1.0/) applies to the data made available in this article, unless otherwise stated. 
catheter. Unfractionated heparin was used for anticoagulation. Platelet count remained normal during this period. His residual renal function declined rapidly, and three months after initiation of dialysis, he was no longer nephrotic. A brachio-axillary arteriovenous graft (AVG) was then created, but he developed four episodes of AVG thrombosis over a period of four months, necessitating repeated thrombectomies and angioplasties. Clopidogrel therapy did not prevent the recurrent thrombosis. Investigations revealed persistently elevated serum homocysteine level of $21.8 \mu \mathrm{mol} / \mathrm{L}$ (reference range 5 to $15 \mu \mathrm{mol} / \mathrm{L}$ ) despite normal folate and vitamin B12 levels. Protein $\mathrm{C}$, protein $\mathrm{S}$, and antithrombin III levels were normal, and he was negative for factor $\mathrm{V}$ Leiden and antiphospholipid antibodies (anti- $\beta 2$ glycoprotein, anti-cardiolipin antibodies and lupus anticoagulant). He had no other history of thrombotic events and the family history was similarly non-contributory. He had never smoked and was not obese (body mass index $20.4 \mathrm{~kg} / \mathrm{m}^{2}$ ).

The patient switched to home hemodialysis, using the NxStage ${ }^{\odot}$ System One cycler, nine months after initiation of renal replacement therapy. His dialysis prescription was based on a target standard urea weekly $\mathrm{Kt} / \mathrm{V}$ of 2.0 and involved five-hour sessions four times a week via his permanent catheter. A single bolus of unfractionated heparin 1,500 units was given at the beginning of each session. The first three sessions were uneventful. At the end of the fourth session, red clots were observed in the dialyzer. Accordingly, the bolus heparin dose was increased to 2,000 units at the following session. During rinse back at the end of the fifth session, white particulate matter measuring 1 to $3 \mathrm{~mm}$ was found firmly adherent to the arterial and venous blood tubing (Figure 1). These were not present in the drip chamber or dialysate lines. Red clots were simultaneously noted in the dialyzer. The patient remained clinically well before, during and immediately after dialysis. The access, effluent and venous pressures during this session were not significantly higher than previous sessions. He presented to our nephrology service six hours later when he noticed decreased thrill in his AVG and was found to have AVG thrombosis, necessitating thrombectomy and stenting. Subsequent dialysis was carried out via conventional hemodialysis using Gambro ${ }^{\circ}$ AK $95^{\mathrm{TM}}$. Unfractionated heparin was given at 1000 units and 500 units per hour as loading and maintenance doses respectively. During rinse back at the end of the next dialysis session, similar white particulate matter was again noted in the tubing, along with red clots in the dialyzer.

Several investigations were performed to elicit the cause of white particulate matter. Blood biochemistry and clotting function were all within acceptable limits. There was a $60 \%$ decrease in his platelet count $\left(405 \times 10^{9} / \mathrm{L}\right)$ two weeks prior; to $163 \times 10^{9} / \mathrm{L}$ on the first day of white thrombi formation. At the second episode two days later, the platelet count increased slightly to $199 \times 10^{9} / \mathrm{L}$. His hemoglobin level $(11.2 \mathrm{~g} / \mathrm{dL})$ and leukocyte count $(8.27 \times$ $\left.10^{9} / \mathrm{L}\right)$ remained stable from baseline. Complement levels were normal. Antibodies for heparin-associated immune thrombocytopenia (HIT) were not measured. Analysis of the dialysate composition was within acceptable limits, with absence of free chlorine, chloramine, heavy metals and endotoxin. Dialysate microbiological cultures were negative. The polyvinylchloride tubing with the white particulate matter was cut and light microscopic examination of the deposits revealed platelet aggregates.

After the second episode of white thrombi formation, he was switched back to NxStage ${ }^{\oplus}$ System One home hemodialysis. An additional midway bolus of heparin 1,000 units was given for a few sessions with no further recurrence of white thrombi. Unfortunately, NxStage ${ }^{\odot}$ home hemodialysis had to be discontinued after two

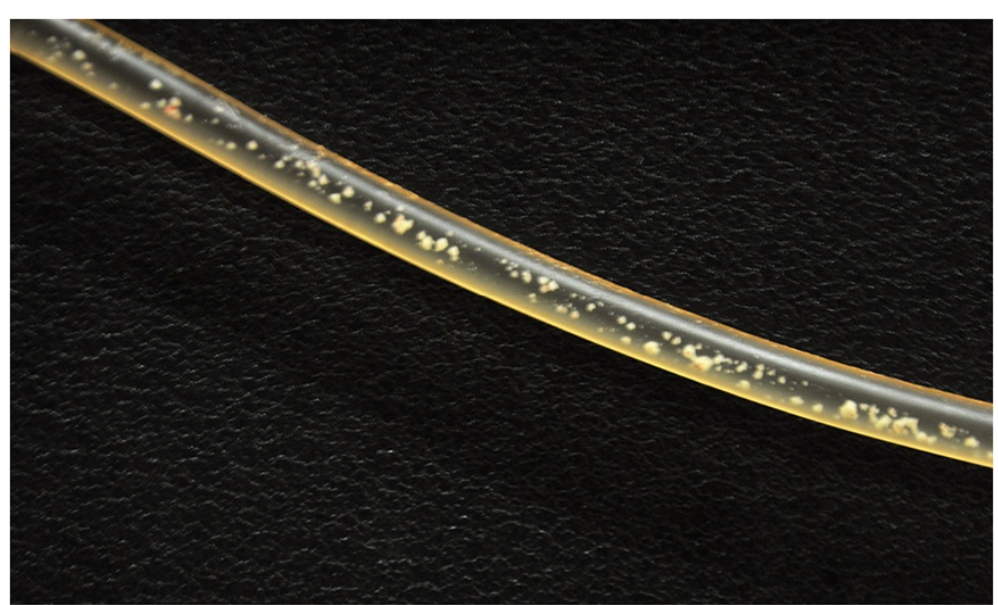

Figure 1 White particulate matter measuring 1 to $3 \mathrm{~mm}$ found adherent to the arterial and venous blood tubing lines after rinse back. 
months as the patient had poor hand dexterity following peripheral nerve injuries sustained during the creation of his AVG. During the 2 months of waiting for neurologic recovery, he was placed on Gambro ${ }^{\circ}$ AK $95^{\text {TM }}$ conventional hemodialysis with standard heparin doses. When he was converted back to NxStage ${ }^{\circ}$ home hemodialysis, he had a newly created arteriovenous fistula. In an attempt to prevent fistula thrombosis, the anticoagulation regimen was changed to intravenous enoxaparin on dialysis days, and subcutaneous enoxaparin on non-dialysis days. His peak anti-Xa levels were maintained at 0.45 to $0.59 \mathrm{IU} / \mathrm{ml}$ on dialysis days. A third episode of white thrombi formation occurred while on NxStage ${ }^{\circ}$ and the dose of enoxaparin was increased with no further recurrence. None of the other nine patients in the hemodialysis unit experienced the same phenomenon throughout the entire period.

\section{Conclusions}

We report the repeated occurrence of white thrombi formation in the hemodialysis tubing of an adolescent male who probably had a pro-thrombotic tendency of undetermined cause. While the appearance of red clots in the dialyzer is a common phenomenon in every hemodialysis unit, the occurrence of white thrombi in the dialysis tubing is relatively rare. These episodes in our patient occurred regardless of the type of dialysis machine or tubing, and appeared to resolve with an increase in heparin dose.

Reports on reactions to hemodialysis tubing lines are scarce. In contrast, reactions to dialyzer membranes have been widely described. Such reactions can cause transient but significant intra-dialytic thrombocytopenia [3-5], and appear to depend on the manufacturer, polyvinylpyrrolidone content and sterilization method of the membrane $[3,6,7]$. Postulated mechanisms include biochemical interactions between blood and dialyzer membrane leading to complement and platelet activation. Activated platelets can form platelet-neutrophil, platelet-erythrocyte and platelet-platelet aggregates during dialysis [3,8]. Despite the formation of such aggregates, reactions to dialyzer membranes do not generally lead to increased risk of thrombosis.

In a previous report by Watnick et al., white thrombi developed in the hemodialysis tubing of 21 out of 34 adult patients in a dialysis unit over a 5 -week period [9]. This phenomenon did not occur after switching to dialysis lines from different manufacturers [9]. The white thrombi, however, recurred immediately after reinstatement of the dialysis lines from the original manufacturer, suggesting that dialysis tubing irregularities sustained during the manufacturing process could be the precipitating cause of white thrombi. These tubing defects could entrap microbubbles and enhance thrombogenicity.
In contrast, our patient was the only one who developed recurrent white thrombi in our hemodialysis unit over the 7-month period, regardless of the type of hemodialysis system (NxStage ${ }^{\circ}$ and $\mathrm{Gambro}^{\circ}$ systems). This supports our conclusion that it was his pro-thrombotic state that predisposed him to white thrombi formation, rather than the properties of any particular dialysis tubing. Similarly, in the series described by Watnick et al., a small subset of patients had recurrent white matter deposits despite the use of different machines on different occasions [9], suggesting the presence of unidentified patient-specific factors such as platelet function abnormalities.

The white thrombi formation described in our patient was most likely related to platelet activation, in view of the significant decrease in platelet count and the presence of platelet aggregates on microscopic examination of the white thrombi. We postulate that there was activation of the coagulation cascade, resulting in generation of thrombin which directly caused platelet activation and aggregation, and ultimately AVG thrombosis. Chronic renal failure is associated with changes in platelet function, coagulation and fibrinolytic factors such as thrombinantithrombin III complex, fibrinopeptide A, D-dimer, von Willebrand factor and beta-thromboglobulin, leading to increased procoagulatory activity [10-13]. In addition, there is release of thromboxane A2 and adenosine diphosphate into the circulation, as well as increased platelet degranulation and aggregation [10-12]. Uremic patients have also been shown to have abnormalities in the platelet glycoprotein GPIb (receptor for von Willebrand factor), which were not corrected by hemodialysis or peritoneal dialysis [13]. Moreover, sera from patients on hemodialysis increased the expression of tissue factor in endothelial cells or smooth muscle cells and enhanced the platelet deposition in extracellular matrix $[14,15]$.

The degree of hyperhomocysteinemia in our patient was expected because of his renal failure [16,17]. Patients on regular hemodialysis have higher serum homocysteine levels than healthy controls [18], possibly due to decreased homocysteine catabolism by the diseased kidneys [19]. Hyperhomocysteinemia is viewed as a non-traditional marker for cardiovascular disease, as it is associated with atherosclerotic events $[18,20]$. While it may be part of the prothrombotic milieu in chronic renal failure [16,17], its exact role in the thrombogenic process is not clear. Possible mechanisms include endothelial cell injury which in turn stimulates the local coagulation system [16,21], or activation of the coagulation cascade via increased plasma tissue factor expression [22].

Our patient developed AVG thrombosis between the first and second episodes of the white thrombi formation, which occurred at consecutive dialysis sessions. While there was no evidence that the AVG thrombosis was directly caused by the white thrombi, it is conceivable that 
the AVG thrombosis was similarly related to the platelet activation. The temporal association of his thrombocytopenia, white clot formation and arterial thrombosis was reminiscent of HIT. Although HIT also involves platelet activation (related to antibodies to heparin-platelet factor 4 (PF4) complex), it was unlikely in our patient since the white thrombi occurred nine months after continuous heparin exposure, way beyond the time period when HIT typically occurs (usually five to ten days after initiation of heparin therapy [23]). For this reason, HIT antibody was not tested in our patient.

An increase in heparin dose appeared to prevent the recurrence of white thrombi in our patient. This was expected since heparin inactivates thrombin and therefore inhibits thrombin-induced activation of platelets. Low molecular weight heparins are probably as effective as unfractionated heparin in preventing extracorporeal circuit thrombosis [24].

In conclusion, this case highlights recurrent white thrombi formation in a dialysis patient with probable pro-thrombotic predisposition of undetermined cause. Such an episode may be a harbinger of AVG thrombosis and may be prevented by an increase in heparin dose.

\section{Consent}

Written informed consent for the publication of this case report and any accompanying images has been obtained from the patient and his father. A copy of the written consent is available for review by the Editor of this journal.

\section{Abbreviations \\ (AVG): Arterio-venous graft; (HIT): Heparin-associated immune \\ thrombocytopenia; (PF4): Heparin-platelet factor 4.}

\section{Competing interests}

The authors declare that they have no competing interests.

\begin{abstract}
Authors' contributions
KPS collated the patient data and drafted the manuscript. WSY supported data collection and writing of the manuscript. IDL supported data collection and writing of the manuscript. SE supported interpretation of data and writing of the manuscript. MA supported interpretation of data and writing of the manuscript. HKY carried out analysis of patient's clinical course, outcomes and interpretation of findings and provided critical review comments for the manuscript. KHN carried out analysis of patient's clinical course, outcomes and interpretation of findings and provided critical review comments for the manuscript. All authors read and approved the final manuscript.
\end{abstract}

\section{Acknowledgments}

We thank Drs Elizabeth Ang, Swasti Chaturvedi and Dimple Rajgor for their assistance in reviewing and editing the manuscript, as well as Ms Karen $J \mathrm{~L}$ Lim and Ms Tay Siew Leng for their assistance in photography.

\section{Received: 13 November 2014 Accepted: 5 January 2015}

Published: 15 January 2015

\section{References}

1. Baccheschi S, Sereni L, De Nitti C, Barbucci R, Tetta C. Blood tubing and cytokine production: effect of sterilization. Ren Fail. 2001;23(3-4):411.
2. Joannidis M, Oudemans-van Straaten HM. Clinical review: Patency of the circuit in continuous renal replacement therapy. Crit Care. 2007;11(4):218

3. Daugirdas JT, Bernardo AA. Hemodialysis effect on platelet count and function and hemodialysis-associated thrombocytopenia. Kidney Int. 2012;82(2):147-157.

4. Hakim R, Schafer A. Hemodialysis-associated platelet activation and thrombocytopenia. Am J Med. 1985;78(4):575.

5. De Prada L, Lee J, Gillespie A, Benjamin J. Thrombocytopenia associated with one type of polysulfone hemodialysis membrane: A report of 5 cases. Am J Kidney Dis. 2013;61(1):131-133.

6. Kiaii M, Djurdjev O, Farah M, Levin A, Jung B, MacRae J. Use of electron-beam sterilized hemodialysis membranes and risk of thrombocytopenia. JAMA. 2011;306(15):1679.

7. Allison S. Dialysis: Dialyzer sterilization method linked to thrombocytopenia. Nat Rev Nephrol. 2011:7(12):676.

8. Sirolli V, Ballone E, Di Stante S, Amoroso L, Bonomini M. Cell activation and cellular-cellular interactions during hemodialysis: effect of dialyzer membrane. Int J Artif Organs. 2002;25(6):529.

9. Watnick S, Stooksbury M, Winter R, Riscoe M, Cohen DM. White thrombus formation in blood tubing lines in a chronic hemodialysis unit. Clin J Am Soc Nephrol. 2008;3(2):382.

10. Sagripanti A, Cupisti A, Baicchi U, Ferdeghini M, Morelli E, Barsotti G. Plasma parameters of the prothrombotic state in chronic uremia. Nephron. 1993;63 (3):273-278.

11. Gordge MP, Leaker BR, Rylance PB, Neild GH. Haemostatic activation and proteinuria as factors in the progression of chronic renal failure. Nephrol Dial Transplant. 1991;6(1):21-26.

12. Martinovic Z, Basic-Jukic N, Pavlovic DB, Kes P. Importance of platelet aggregation in patients with end-stage renal disease. Acta Clin Croat. 2013;52(4):472-477.

13. Salvati F, Liani M. Role of platelet surface receptor abnormalities in the bleeding and thrombotic diathesis of uremic patients on hemodialysis and peritoneal dialysis. Int J Artif Organs. 2001;24(3):131-135.

14. Serradell M, Diaz-Ricart M, Cases A, Zurbano MJ, Aznar-Salatti J, Lopez-Pedret J, et al. Uremic medium disturbs the hemostatic balance of cultured human endothelial cells. Thromb Haemost. 2001;86(4):1099-1105.

15. Chitalia VC, Shivanna S, Martorell J, Balcells M, Bosch I, Kolandaivelu K, et al. Uremic serum and solutes increase post-vascular interventional thrombotic risk through altered stability of smooth muscle cell tissue factor. Circulation. 2013;127(3):365-376.

16. Farid FA, Faheem MS, Heshmat NM, Shaheen KY, Saad SS. Study of the homocysteine status in children with chronic renal failure. Am J Nephrol. 2004;24(3):289-295.

17. Fadel Fl, Elshamaa MF, Essam RG, Elghoroury EA, El-Saeed GS, El-Toukhy SE, et al. Some amino acids levels: glutamine, glutamate, and homocysteine, in plasma of children with chronic kidney disease. Int J Biomed Sci. 2014;10 (1):36-42.

18. Wu CC, Zheng CM, Lin YF, Lo L, Liao MT, Lu KC. Role of homocysteine in end-stage renal disease. Clin Biochem. 2012;45(16-17):1286-1294.

19. Bostom AG, Lathrop L. Hyperhomocysteinemia in end-stage renal disease: prevalence, etiology, and potential relationship to arteriosclerotic outcomes. Kidney Int. 1997;52(1):10-20.

20. Van Campenhout A, Moran CS, Parr A, Clancy P, Rush C, Jakubowski H, et al. Role of homocysteine in aortic calcification and osteogenic cell differentiation. Atherosclerosis. 2009;202(2):557-566.

21. Tsai JC, Perrella MA, Yoshizumi M, Hsieh CM, Haber E, Schlegel R, et al. Promotion of vascular smooth muscle cell growth by homocysteine: a link to atherosclerosis. Proc Natl Acad Sci U S A. 1994;91(14):6369-6373.

22. Cetin O, Bekpinar S, Unlucerci Y, Turkmen A, Bayram C, Ulutin T. Hyperhomocysteinemia in chronic renal failure patients: relation to tissue factor and platelet aggregation. Clin Nephrol. 2006;65(2):97-102.

23. Syed S, Reilly R. Heparin-induced thrombocytopenia: a renal perspective. Nat Rev Nephrol. 2009;5(9):501

24. Lim W, Cook DJ, Crowther MA. Safety and efficacy of low molecular weight heparins for hemodialysis in patients with end-stage renal failure: a meta-analysis of randomized trials. J Am Soc Nephrol. 2004;15(12):3192-3206.

doi:10.1186/1471-2369-16-3

Cite this article as: Sathe et al:: Recurrent white thrombi formation in hemodialysis tubing: a case report. BMC Nephrology 2015 16:3. 